\title{
Setting research priorities for
} global respiratory medicine within the National Institute for Health Research (NIHR) Global Health Research Unit in Respiratory Health (RESPIRE)

Igor Rudan¹, Dhiraj Agrawal' ${ }^{2}$, Norita Hussein ${ }^{3}$, Ai Theng Cheong ${ }^{4}$, Steve Cunningham ${ }^{5}$, David Dockerell ${ }^{6}$, Sazlina Shariff Ghazali ${ }^{4}$, Deesha Ghorpade ${ }^{7}$, Monsur Habib $^{8}$, Tabish Hazir ${ }^{9,10}$, Sanjay Juvekar ${ }^{2}$, Anand Kawade ${ }^{2}$, Ping Yein Lee ${ }^{4}$, Su May Liew $^{3}$, Saturnino Luz ${ }^{1}$, Ee Ming Khoo ${ }^{3}$, Harish Nair ${ }^{1}$, John Norrie ${ }^{1}$, Rutuja Patil ${ }^{2}$, Hilary Pinnock ${ }^{1}$, Siti Nurkamilla Ramdzan ${ }^{3}$, Sudipto Roy ${ }^{2}$, Hani Salim ${ }^{4}$, Pam Smith ${ }^{11}$, Hana Mahmood Yahya ${ }^{10}$, Siân Williams ${ }^{12}$, Harry Campbell ${ }^{1}$, Aziz Sheikh ${ }^{1}$

\footnotetext{
${ }^{1}$ Usher Institute, The University of Edinburgh, Edinburgh, UK

${ }^{2}$ Vadu Rural Health Program, King Edward Memorial Hospital Research Centre (KEMHRC) Pune, India

${ }^{3}$ Department of Primary Care Medicine, Faculty of Medicine, University of Malaya, Malaysia

${ }^{4}$ Department of Family Medicine, University Putra, Malaysia

${ }^{5}$ Child Life and Health, The University of Edinburgh, Edinburgh, UK

${ }^{6}$ MRC Centre for Inflammation Research, The University of Edinburgh, Edinburgh, UK

${ }^{7}$ Chest Research Foundation, India

${ }^{8}$ Bangladesh Primary Care Respiratory Society

${ }^{9}$ Pakistan Institute of Medical Sciences

${ }^{10}$ Maternal Neonatal and Child Health Research Network (MNCHRN), Pakistan

${ }^{11}$ Nursing Edinburgh, Edinburgh, UK

${ }^{12}$ International Primary Care Respiratory Group, Edinburgh, UK
}

$\mathrm{T}$ he NIHR Global Health Research Unit in Respiratory Health (henceforth 'RESPIRE') is a research and academic capacity development initiative funded by the UK Government through its National Institute of Health Research (NIHR). RESPIRE's focus is to undertake applied respiratory health in both communicable and non-communicable disorders, which has the potential to improve the respiratory health of children and adults in Bangladesh, India, Malaysia and Pakistan [1]. RESPIRE's working ethos is to work bottom-up to undertake research priorities that have been identified by RESPIRE investigators in our partner low- and middle-income countries (LMICs) [1]. To this end, we conducted an internal process of identifying research priorities within the RESPIRE collaboration using the Child Health 
The NIHR Global Health Research Unit in Respiratory Health ('RESPIRE') is a research and academic capacity development initiative funded by the UK Government through its National Institute of Health Research (NIHR). We conducted an internal process of identifying research priorities within the RESPIRE collaboration using the Child Health and Nutrition Research Initiative's (CHNRI) method. A total of 35 RESPIRE investigators submitted 37 research ideas. The scoring of those ideas was then performed by 26 (out of 65 invited) RESPIRE researchers based on 10 pre-agreed criteria: answerability, feasibility, effectiveness, applicability, affordability, potential for cross-country scalability, burden size, equity, safety and sustainability.

and Nutrition Research Initiative's (CHNRI) method [2]. The outcomes of this process were then used, in conjunction with other approaches, to develop the list of the research projects led by RESPIRE investigators that would receive financial support from the RESPIRE budget [2].

\section{THE CHNRI EXERCISE TO IDENTIFY RESEARCH PRIORITIES WITHIN RESPIRE}

We first asked investigators from the RESPIRE collaboration to submit their ideas for research projects and PhD projects, expecting that those ideas would mainly be generated within Bangladesh, India, Malaysia and Pakistan, in line with their local needs. We then conducted an exercise in assessment of all submitted ideas using the CHNRI method, described briefly in the Box 1 [3-11]. A total of 35 investigators who were named on the RESPIRE research grant proposal submitted 37 research ideas. We then prepared those ideas for scoring and invited both the 35 proposers and a further 30 members from their academic teams to take part in the process of scoring ideas. The scoring was based on 10 criteria, which were agreed in advance by the RESPIRE team during their first meeting in Edinburgh in June 2017. These criteria were:

1. Answerability: is this research question likely to be answered by this research using the proposed methods and approaches?

2. Feasibility: is this research question likely to lead to deliverable outcomes over the time scale of this project?

3. Effectiveness: is this research question likely to lead to interventions that will effectively reduce disease burden, change provision of care, change policy or practice?

Box 1. The CHNRI method for setting research priorities

The CHNRI method uses the principle of crowdsourcing to score proposed research ideas against a pre-defined set of criteria. This enables funders and policymakers to view the strengths, the weaknesses and relative ranking of each proposed research idea, based on submitted opinions of a larger number of experts. This method uses a systematic, transparent, and democratic approach to priority setting. While it allows researchers to independently generate and score research questions (RQs), it also involves funders, policymakers, and other stakeholders at an early stage of the process, ensuring their ownership in the outcomes. The CHNRI method has thus far been implemented in about 100 studies led by multilateral organisations (eg, World Health Organization, United Nations International Children's Emergency Fund (UNICEF)), national governments (eg, China, India, Iran, South Africa), and funders (eg, The Bill and Melinda Gates Foundation) to set research priorities in areas ranging from the reduction of global child mortality, dementia, or disability to the efficient execution of national health plans. The recognised advantages of this method include its systematic nature, transparency and replicability, clearly defined context and criteria, involvement of the funders, stakeholders and policy makers, a structured way of obtaining information, informative and intuitive quantitative outputs, studying the level of agreement over each proposed research idea, and independent scoring of many experts, thus limiting the influence of individuals on the rest of the group [2-11]. 
4. Applicability: is this research question translational in nature, in accordance to RESPIRE's overall aims and objectives?

5. Affordability: is this research question affordable within the context and a good value for money in the way it is proposed?

6. Potential for cross-country scalability: is this research question scalable to other populations, providing an opportunity to be conducted/adapted in several of our partner countries?

7. Burden size: does this research question address a significant healthcare problem and/or disease burden?

8. Equity: is this research question likely to reduce inequity?

9. Safety: is this research question likely to have any harmful or unintended consequences?

10. Sustainability: would this research question create data or resources that will lead to opportunities for further, sustainable funding?

The contextual background to guide the scoring was defined in terms of space, time, population of interest and disease burden of interest, as is standard practice in the CHNRI process [3,4,11]. Space was defined as the four partner countries (ie, Bangladesh, India, Malaysia and Pakistan), the time as the interval between now and year 2025, the population of interest was defined as respiratory disease sufferers, and the disease burden of interest was defined as "all respiratory diseases" within the defined space, time and population.

The scorers were also instructed to think beyond the endpoints of research questions and to keep in mind their broader scope and relevance. Assessing some of the proposed research questions was only possible if thinking was shifted from purely assessing the likelihood that the proposed research would achieve their endpoints, to what those endpoints could mean in the wider context and how these could help improve the overall current situation in terms of risk avoidance and intervention coverage at the level of the four partner countries in 2018.

A total of 26 RESPIRE researchers (among the 65 invited) returned their scores by the stated deadline and this allowed us to conduct the analysis of their input, which was performed in line with the guidelines for implementation of the CHNRI process [11]. The scorers were asked to assess each proposed research idea according to the 10 questions posed above as "yes" (coded as 1 point), "no" (0 points), "not sure" (0.5 points) or "don't know" (input left blank). The received scores allowed computation of "research priority scores" for each criterion and the overall priority score, the latter being used for the final ranking of the proposed research questions (Table 1) $[3,4,11]$. In addition, the "average expert agreement" (AEA) was computed for each proposed research question to demonstrate the level of controversy related to each proposed research question among the scorers who took part in the CHNRI exercise [3,4,11].

The highest-ranked research ideas included proposals to measure the burden of chronic obstructive pulmonary disease (COPD) in the four RESPIRE partner countries (ie, Bangladesh, India, Malaysia and Pakistan), along with the development of a protocol for a COPD prevalence study; also, HEAL-ASTHMA studies, a cross-sectional study to determine the prevalence of limited health literacy followed by a quasi-experimental feasibility study of a pictorial action plan for asthma in Malaysia; then, implementation of pulse oximetry as a point-of-care diagnostic in health facilities that provide integrated management of childhood illness; assessing the feasibility of using a well-established tele-consultation facility (Micro-Health Centre - MHC) in management of COPD and asthma in a resource constrained remote rural area; and studying the perception of under-five pneumonia among caregivers of selected rural and semi urban communities of Pakistan through a mixed methodology-based study. The outcomes of this process were then used, in conjunction with other approaches, to develop the list of the research projects led by RESPIRE investigators that would receive financial support from the RESPIRE budget. 
Table 1. The final rank of 37 proposed research questions that relate to global respiratory health and that could be conducted within the RESPIRE programme*

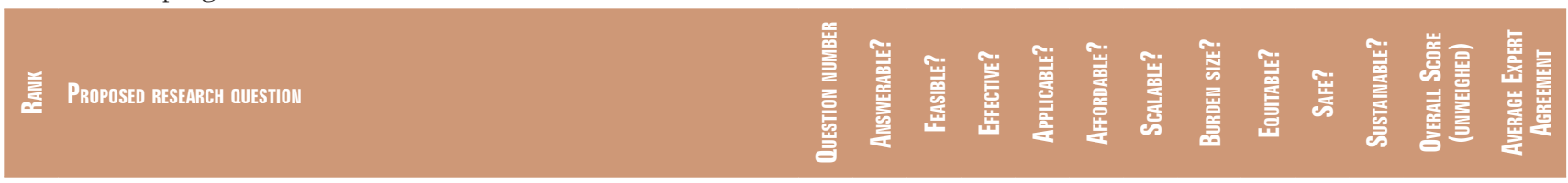

1 Measuring the burden of COPD in the four partner countries $\quad \begin{array}{llllllllllllll}15 & 94 & 93 & 83 & 85 & 93 & 96 & 98 & 92 & 95 & 91 & 91.9 & 0.804\end{array}$

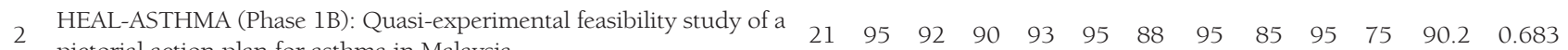

2 pictorial action plan for asthma in Malaysia

3 Implementation of pulse oximetry as a point-of-care diagnostic in IMCI

health facilities

4 Development of a protocol for a COPD prevalence study in India, Bangladesh, Pakistan and Malaysia

Assessing the feasibility of using a well-established teleconsultation fa-

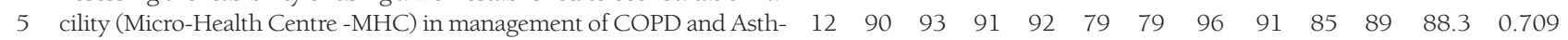
ma in a resource constrained remote rural area

Perception of under-five pneumonia among caregivers of selected rural

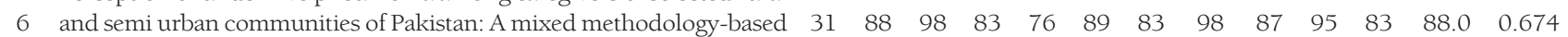
study

7 HEAL-ASTHMA (Phase 1A): A cross-sectional study to determine the

7 prevalence of limited health literacy

$\begin{array}{lllllllllllll}20 & 95 & 95 & 83 & 73 & 92 & 83 & 100 & 93 & 92 & 67 & 87.3 & 0.674\end{array}$

8 Haze project in Malaysia: reporting haze and studying respiratory ef-

fects

$\begin{array}{lllllllllllll}24 & 81 & 88 & 92 & 92 & 86 & 73 & 96 & 91 & 92 & 79 & 87.0 & 0.404\end{array}$

9 Developing and piloting an ICT-based intervention for adult asthma

with limited health literacy to improve asthma self-management

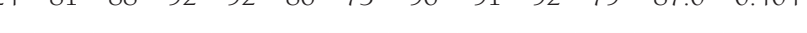

10 Setting up a registry for asthma to measure the prevalence of asthma 10 in adults in Malaysia

$\begin{array}{lllllllllllll}7 & 98 & 82 & 83 & 94 & 86 & 80 & 98 & 78 & 89 & 82 & 86.9 & 0.683\end{array}$

Community health worker driven COPD awareness and screening pro-

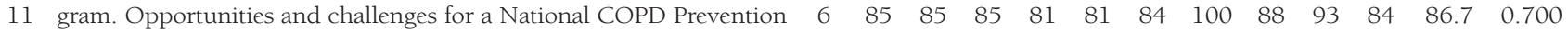
and Control Program.

Care-seeking practices of and barriers to; care-seeking for pneumonia

12 in children aged less than five years in tribal and non-tribal rural areas $\quad \begin{array}{lllllllllllll}38 & 92 & 98 & 87 & 83 & 88 & 66 & 93 & 90 & 93 & 76 & 86.6 & 0.700\end{array}$ of Pune district, India

$\begin{array}{lllllllllllllll} & \begin{array}{l}\text { Palliative care needs of patients with chronic obstructive pulmonary } \\ \text { disease }\end{array} & 3 & 90 & 89 & 83 & 79 & 90 & 87 & 91 & 79 & 93 & 82 & 86.5 & 0.700\end{array}$

14 Culturally Tailored School-Based Complex Interventions for Child-

4 hood Asthma in Malaysia (cut-asthma): An Implementation Study

Community health worker driven COPD awareness and screening pro-

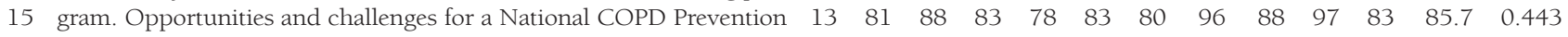
and Control Program

Developing and pilot-testing the effectiveness of a culturally tailored

16 awareness and supported self-management intervention for patients $17 \quad \begin{array}{lllllllllllll}17 & 83 & 78 & 82 & 88 & 91 & 82 & 95 & 86 & 88 & 83 & 85.4 & 0.613\end{array}$ with COPD

17 Long-term respiratory morbidity (asthma) in children with RSV in the

neonatal period- follow up of ANISA Cohort in Sylhet

$\begin{array}{lllllllllllll}35 & 86 & 72 & 82 & 78 & 71 & 85 & 97 & 88 & 91 & 88 & 83.8 & 0.487\end{array}$

8 Enhancing access to pulmonary rehabilitation (PR) through implemen-

8 tation research in Bangladesh

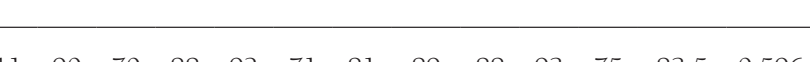

Evaluation of mobile phone (M- Health) technology for self manage-

19 ment in pollen asthma patients in Pakistan

$\begin{array}{lllllllllllll}11 & 90 & 70 & 88 & 92 & 71 & 81 & 89 & 88 & 93 & 75 & 83.5 & 0.596\end{array}$

20 Reliability and validity of asthma control test among Chinese, Malays

20 and Indians in Malaysia

21 Trial of pulmonary rehabilitation

$\begin{array}{lllllllllllll}10 & 88 & 80 & 81 & 88 & 82 & 73 & 90 & 75 & 91 & 86 & 83.4 & 0.617\end{array}$

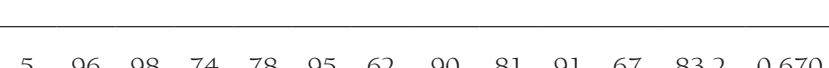

Feasibility of using m-health technology to counsel caregivers of chil-

22 dren under five on prevention against pneumonia and improved care $\quad \begin{array}{llllllllllllll}36 & 77 & 79 & 85 & 90 & 74 & 81 & 92 & 79 & 84 & 80 & 82.1 & 0.630\end{array}$ seeking through lady health workers in rural communities

23 Long-term respiratory morbidity (asthma) in children with RSV in the neonatal period- follow up of ANISA cohort in 3 South Asian countries

24 Factors responsible for uncontrolled asthma in a rural community in

24 Southern India

25 Integrating childhood asthma detection and management into the primary health care in rural Pune district

\begin{tabular}{llllllllllllll}
5 & 96 & 98 & 74 & 78 & 95 & 62 & 90 & 81 & 91 & 67 & 83.2 & 0.670 \\
25 & 81 & 77 & 83 & 92 & 85 & 72 & 82 & 77 & 93 & 81 & 82.2 & 0.448 \\
\hline 36 & 77 & 79 & 85 & 90 & 74 & 81 & 92 & 79 & 84 & 80 & 82.1 & 0.630 \\
34 & 87 & 72 & 82 & 80 & 63 & 82 & 94 & 79 & 94 & 85 & 81.8 & 0.535 \\
9 & 82 & 81 & 78 & 73 & 87 & 70 & 98 & 83 & 91 & 73 & 81.6 & 0.643 \\
22 & 83 & 79 & 83 & 85 & 82 & 77 & 85 & 85 & 93 & 64 & 81.5 & 0.570 \\
16 & 80 & 74 & 79 & 90 & 72 & 78 & 95 & 85 & 91 & 72 & 81.5 & 0.517
\end{tabular}

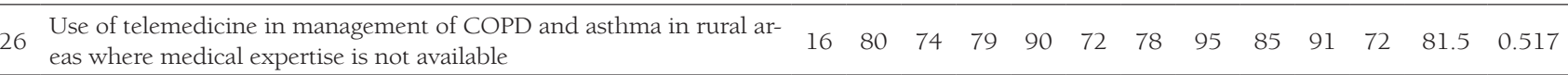


Table 1. Continued

\begin{tabular}{|c|c|c|c|c|c|c|c|c|c|c|c|c|c|c|}
\hline 差 & PROPOSED RESEARCH QUESTION & 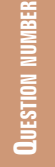 & 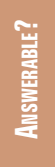 & 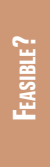 & 冚 & 总 & 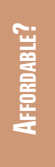 & 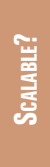 & 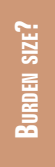 & 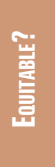 & 蓆 & 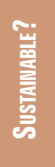 & 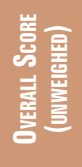 & 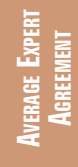 \\
\hline 27 & $\begin{array}{l}\text { Exploration of pneumonia-related policy formation and implementa- } \\
\text { tion in Pakistan }\end{array}$ & 30 & 91 & 89 & 85 & 64 & 83 & 68 & 89 & 81 & 92 & 71 & 81.5 & 0.526 \\
\hline 28 & Development of spirometry predictive values for Indian population & 1 & 86 & 90 & 77 & 78 & 84 & 72 & 89 & 71 & 91 & 76 & 81.4 & 0.639 \\
\hline 29 & $\begin{array}{l}\text { Feasibility of using family psychoeducation for pneumonia prevention } \\
\text { among caregivers of children under five }\end{array}$ & 37 & 81 & 79 & 78 & 79 & 87 & 71 & 95 & 88 & 87 & 64 & 80.7 & 0.509 \\
\hline 30 & $\begin{array}{l}\text { Assessment of ASHA's (accredited social health activist) workload and } \\
\text { its determinants }\end{array}$ & 4 & 94 & 93 & 74 & 73 & 88 & 65 & 70 & 84 & 93 & 69 & 80.3 & 0.648 \\
\hline 31 & $\begin{array}{l}\text { Implementation of bubble CPAP in the management of severe pneu- } \\
\text { monia in young children in secondary level health facilities }\end{array}$ & 33 & 93 & 89 & 90 & 82 & 74 & 70 & 87 & 64 & 65 & 89 & 80.2 & 0.517 \\
\hline 32 & $\begin{array}{l}\text { Community use of digital auscultation to improve diagnosis of paedi- } \\
\text { atric pneumonia in Sylhet, Bangladesh }\end{array}$ & 26 & 85 & 80 & 81 & 79 & 76 & 81 & 90 & 68 & 79 & 75 & 79.4 & 0.504 \\
\hline 33 & $\begin{array}{l}\text { Prevention, detection and treatment of adult lung disease (with a focus } \\
\text { on lung cancer) in a poor, rural population in Tamil Nadu }\end{array}$ & 18 & 71 & 60 & 68 & 76 & 84 & 69 & 94 & 85 & 94 & 64 & 76.8 & 0.474 \\
\hline 34 & $\begin{array}{l}\text { Lung cancer and chronic respiratory disease: Development and pilot } \\
\text { testing of an intervention in a southern Indian rural community }\end{array}$ & 14 & 75 & 64 & 76 & 83 & 69 & 69 & 93 & 84 & 90 & 60 & 76.4 & 0.526 \\
\hline 35 & $\begin{array}{l}\text { Documenting pneumonia case management practices in selected com- } \\
\text { munities in Pakistan: a qualitative study }\end{array}$ & 29 & 83 & 93 & 68 & 63 & 83 & 60 & 84 & 85 & 84 & 56 & 75.9 & 0.474 \\
\hline 36 & Allergen mapping and seasonal exacerbations of asthma in Pakistan & 23 & 78 & 76 & 72 & 75 & 64 & 47 & 83 & 70 & 93 & 61 & 72.0 & 0.391 \\
\hline 37 & $\begin{array}{l}\text { Construction of a computational framework to automatically interpret } \\
\text { chest x-rays and diagnose pneumonia }\end{array}$ & 28 & 69 & 68 & 64 & 70 & 71 & 75 & 83 & 72 & 72 & 68 & 71.2 & 0.443 \\
\hline
\end{tabular}

*Their research priority scores are shown for each of the 10 priority-setting criteria. The overall research priority score, which provides the basis for the final ranking, is also presented, along with the average expert agreement. Detailed explanations of these measures are provided in references [4,8-11].

\section{OUTCOMES}

Table 1 provides the final rankings for the 37 proposed research questions that relate to global respiratory health and are of interest to the RESPIRE initiative. Research priority scores for these ideas are shown for each of the 10 priority-setting criteria and they are self-explanatory, as well as the overall research priority score, which is the basis for the final ranking.

Research ideas with the highest scores included proposals to measure the burden of COPD in the four partner countries, along with the development of a protocol for a COPD prevalence study; also, HEAL-ASTHMA studies, a cross-sectional study to determine the prevalence of limited health literacy followed by a quasi-experimental feasibility study of a pictorial action plan for asthma in Malaysia; then, implementation of pulse oximetry as a point-of-care diagnostic in health facilities that provide integrated management of childhood illness; assessing the feasibility of using a well-established tele-consultation facility (Micro-Health Centre -MHC) in management of COPD and Asthma in a resource constrained remote rural area; and studying the perception of under-five pneumonia among caregivers of selected rural and semi urban communities of Pakistan through a mixed-methods study.

Most importantly, all projects received an overall research priority score greater than 70.0 , while a typical range of scores in CHNRI exercises is between 27.0-91.0 [4]. This means that the 26 RESPIRE investigators felt that all 35 proposed research ideas were promising, had few apparent shortcomings and were worth doing. However, there were rather strong internal differences in opinions related to 8 out of the 37 proposed projects, as captured by $A E A<50.0$ (Table 1), which meant that some of the proposed ideas were probably more controversial than others.

There are also some specific concerns related to some proposed research questions in relation to particular criteria. The criteria around which most concerns were raised, as captured by the criterion-specific research score $<70.0$, were sustainability (in 9 cases), scalability (in 7 cases) and effectiveness (in 3 cases; see Table 1). 


\section{CONCLUSIONS}

The CHNRI exercise worked very well within the RESPIRE initiative and gave support to all proposed research ideas, while highlighting some specific topics that warranted further discussions. It served the purpose of quickly assessing strengths and weaknesses of the proposed research ideas that we believed could help reduce respiratory disease burden in partner countries by 2025. This was in line with recommendations from RESPIRE's International Steering Committee, which recommended that prioritisation takes place to allow for partner input into funding allocation decisions.

The CHNRI process provided a useful complementary insight to that provided by the peer-review process into perceived strengths and weaknesses of each proposed research project and $\mathrm{PhD}$ project. It informed the prioritisation of funding for large RESPIRE projects, smaller projects and PhD projects, in addition to the Panel discussion in Dhaka in February 2018 and the external peer review. Its results showed a rather narrow range in the overall research priority scores, ie, between 71.2 and 91.9 . We believe that this is primarily a result of very well explained context and rules of the CHNRI project to all participants in the exercise during the first RESPIRE meeting in Edinburgh in June 2017. This led to an initial selection of a relatively small number of research ideas, all of which arose from the reality of the contexts of the partner countries as real needs. All of the proposed projects had a reasonable chance of implementation and success in generating new knowledge, and this was reflected in the overall scores. Typical CHNRI exercises can usually have up to 10 times more research ideas proposed by a much larger groups of researchers, and many of their ideas may well be quite speculative and risky, leading to a much wider dispersion of the overall research priority scores.

The process was not free from some limitations. Primarily, scorers in the CHNRI exercise were not independent from the experts who set the topics. Also, in a sample size of 37 larger sampling errors can be expected. After the exercise was conducted, some of the scorers commented that more detail could have been provided to some of the proposed ideas, as this could have affected their scores. Some ideas were changing over the period of the CHNRI process as they were being developed. Therefore, it is possible that some scorers may have missed the most recent update on some ideas (eg, if they couldn't attend the latest RESPIRE teleconference). There were also comments from the scorers that a subsequent, larger exercise could be considered. Such exercise would include more ideas, scorers and a longer time frame. Future exercises could also be improved if they considered not only opinions from scorers, but also their confidence in their opinions. Such an approach has been shown to increase the validity of exercises which use crowdsourcing to derive collective opinion [12-14].

\footnotetext{
Acknowledgements: We thank all RESPIRE investigators for their contribution to useful discussions on research
priorities in global respiratory medicine, which took place in Dhaka, Bangladesh, on 7th-9th February 2018.
Disclaimer: This research was commissioned by the National Institute of Health Research using Official Devel-
opment Assistance (ODA) funding. The views expressed are those of the author(s) and not necessarily those of
the NHS, the NIHR or the Department of Health and Social Care
Funding: United Kingdom's National Institute of Health Research (NIHR) through Global Challenges Research
Fund (GCRF)
Authorship contributions: IR designed the study, prepared the instructions and scoring sheets, invited the input
from RESPIRE technical experts, analysed the results and drafted the paper. DA, NB, ATC, SC, DD, SSG, DG, MH,
TH, SJ, AK, PYL, SML, SL, KEM, HN, RP, HP, SNR, SR, HS, PS, HMY, SW and HC contributed their input to the
scoring process, provided useful comments during the Dhaka meeting and checked the paper for the important
intellectual content. JN, HC and AS contributed to study design and writing of the final version of the paper.
Competing interests: The authors have completed the Unified Competing Interest form at www.icmje.org/coi_-
disclosure.pdf (available on request from the corresponding author) and declare no conflict of interest.
} 
1 Sheikh A, Campbell H, Balharry D, Baqui AH, Bogaert D, Cresswell K, et al. RESPIRE: The National Institute of Health Research's (NIHR) Global Respiratory Health Unit. J Glob Health. 2018;8:020101._doi:10.7189/jogh.08.020101

2 Rudan I. Global health research priorities: mobilizing the developing world. Public Health. 2012;126:237-40. Medline:22325672 doi:10.1016/j.puhe.2011.12.001

3 Yoshida S. Approaches, tools and methods used for setting priorities in health research in the 21(st) century. J Glob Health. 2016;6:010507. Medline:26401271 doi:10.7189/jogh.06.010507

4 Rudan I, Yoshida S, Chan KY, Sridhar D, Wazny K, Nair H, et al. Setting health research priorities using the CHNRI method: VII. A review of the first 50 applications of the CHNRI method. J Glob Health. 2017;7:011004. Medline:28685049 doi:10.7189/jogh.07.011004

5 Rudan I, Yoshida S, Chan KY, Cousens S, Sridhar D, Bahl R, et al. Setting health research priorities using the CHNRI method: I. Involving funders. J Glob Health. 2016;6:010301. Medline:26401269 doi:10.7189/jogh.06.010301

6 Yoshida S, Cousens S, Wazny K, Chan KY. Setting health research priorities using the CHNRI method: II. Involving researchers. J Glob Health. 2016;6:010302. Medline:27350870 doi:10.7189/jogh.06.010302

7 Yoshida S, Wazny K, Cousens S, Chan KY. Setting health research priorities using the CHNRI method: III. Involving stakeholders. J Glob Health. 2016;6:010303. Medline:27303649 doi:10.7189/jogh.06.010303

8 Rudan I. Setting health research priorities using the CHNRI method: IV. Key conceptual advances. J Glob Health. 2016;6:010501. Medline:27418959 doi:10.7189/jogh.06.010501

9 Rudan I, Yoshida S, Wazny K, Chan KY, Cousens S. Setting health research priorities using the CHNRI method: V. Quantitative properties of human collective knowledge. J Glob Health. 2016;6:010502. Medline:27350873 doi:10.7189/ jogh.06.010502

10 Yoshida S, Rudan I, Cousens S. Setting health research priorities using the CHNRI method: VI. Quantitative properties of human collective opinion. J Glob Health. 2016;6:010503. Medline:27350874 doi:10.7189/jogh.06.010503

11 Rudan I, Gibson JL, Ameratunga S, El Arifeen S, Bhutta ZA, Black M, et al; Child Health and Nutrition Research Initiative. Setting priorities in global child health research investments: guidelines for implementation of CHNRI method. Croat Med J. 2008;49:720-33. Medline:19090596 doi:10.3325/cmj.2008.49.720

12 Koriat A. When are two heads better than one and why? Science. 2012;336:360-2. Medline:22517862 doi:10.1126/ science. 1216549

13 Hertwig R. Psychology. Tapping into the wisdom of the crowd - with confidence. Science. 2012;336:303-4. Medline:22517847 doi:10.1126/science. 1221403

14 Surowiecki J. The wisdom of crowds. New York: Random House; 2004.

\section{Correspondence to:}

Professor Igor Rudan

Chair in International Health and Molecular Medicine Joint Director, Centre for Global Health Research and WHO Collaborating Centre for Population Health Research and Training

Director of Research, The Usher Institute for Population Health Sciences and Informatics

The University of Edinburgh

MacKenzie House, 30 West Richmond Street

Edinburgh EH8 9DX, Scotland, UK

Igor.Rudan@ed.ac.uk 